\title{
Low energy X-ray grating interferometry at the Brazilian Synchrotron
}

\author{
F.J. Koch ${ }^{\mathrm{a}}$, F.P. O’Dowd ${ }^{\mathrm{b}}$, M.B. Cardoso ${ }^{\mathrm{b}}$, R.R. Da Silva ${ }^{\mathrm{c}}$, M. Cavicchioli ${ }^{\mathrm{c}}$, S.J.L. Ribeiro ${ }^{\mathrm{c}}$, \\ T.J. Schröter ${ }^{\mathrm{a}}$, A. Faisal ${ }^{\mathrm{a}}$, P. Meyer ${ }^{\mathrm{a}}$, D. Kunka ${ }^{\mathrm{a}}$, J. Mohr ${ }^{\mathrm{a}, *}$ \\ a Institute of Microstructure Technology, Karlsruhe Institute of Technology, Hermann-von-Helmholtz-Platz 1, 76344 Eggenstein-Leopoldshafen, Germany \\ b Brazilian Synchrotron Light Laboratory, 13083-970 Campinas, Brazil \\ c Institute of Chemistry - São Paulo State University - UNESP, CP 355, Araraquara, SP 14801-970, Brazil
}

\section{A R T I C L E I N F O}

Keywords:
X-ray
Optics
Grating interferometry
Nanocrystals

\begin{abstract}
A B S T R A C T
Grating based X-ray differential phase contrast imaging has found a large variety of applications in the last decade. Different types of samples call for different imaging energies, and efforts have been made to establish the technique all over the spectrum used for conventional X-ray imaging. Here we present a two-grating interferometer working at $8.3 \mathrm{keV}$, implemented at the bending magnet source of the IMX beamline of the Brazilian Synchrotron Light Laboratory. The low design energy is made possible by gratings fabricated on polymer substrates, and makes the interferometer mainly suited to the investigation of light and thin samples. We investigate polymer microspheres filled with $\mathrm{Fe}_{2} \mathrm{O}_{3}$ nanoparticles, and find that these particles give rise to a significant visibility reduction due to small angle scattering.
\end{abstract}

\section{Introduction}

X-ray differential phase contrast imaging based on the Talbot effect (X-ray Talbot Interferometry - XTI) is a widely used technique both at synchrotron and laboratory X-ray sources [1,2]. Along with the conventional transmission image of a sample, it provides both a differential phase contrast and a dark field image of the sample. The technique is of particular interest for the investigation of light materials, as for low $\mathrm{Z}$ elements, the real part $\delta$ of the refractive index $n=1-\delta+i \beta$, can be three orders of magnitude greater than the imaginary part $\beta$ [3]. Applications range from detecting diseases in different body parts such as the lung or breast $[4,5]$ to food screening and materials analysis such as tracking water transport in cement $[6,7]$. The type of sample under investigation determines the optimal photon energy for imaging. Grating interferometry has been shown to work at photon energies as high as $180 \mathrm{keV}$ (monochromatic beam) [8] and as low as $8 \mathrm{keV}$ (Copper K- $\alpha$ line) [9]. In general, i.e. neglecting absorption edges, both $\delta$ and $\beta$ are higher for lower photon energies, yielding a larger contrast. However, the figure of merit is the signal to noise ratio (SNR) [10,11]. Determining the energy with the highest SNR in multimodal imaging is not trivial, as the individual modalities exhibit different optima. For the case of XTI, the noise $\sigma$ is dominated by the photon count on the detector $N$ and thus exponentially increases with sample thickness $z, \sigma^{2} \propto 1 / N=1 / N_{0} \exp (-2 k \beta z)$ [12]. Here, $N_{\boldsymbol{O}}$ denotes the number of photons incident on the sample and $k$ is the wavenum- ber. A good proxy of the phase contrast signal is the phase shift $\Delta \Phi=k \delta z$ introduced by a sample, which is linearly dependent on its thickness $z$. The SNR for the phase image is thus proportional to $S N R \propto k \delta z \bullet \exp (-k \beta z)$, and depends strongly on sample material and thickness. As an example, the maximum of the function for a $200 \mu \mathrm{m}$ silica sample (e.g. a microsphere as used in many applications) is at $10.6 \mathrm{keV}$, while a polyethylene terephthalate (PET) sample of the same thickness has its maximum at $5.3 \mathrm{keV}$. With increasing thickness, the maxima are shifted to higher energy. These calculations, based on data from [13], show that the ideal photon energy for some relevant samples lies below $15 \mathrm{keV}$. In the dark field modality, the choice of energy has another implication: it determines, together with the geometrical dimensions of the setup, the autocorrelation length that is probed within the sample, and thus the microstructural sizes to which the dark field signal is most sensitive [14].

Unfortunately, the current technology for grating fabrication poses limits for grating interferometry at low $(<15 \mathrm{keV})$ design energy. At $8 \mathrm{keV}$, the lowest photon energy reported in grating based interferometry so far [9], a gold thickness of $6.5 \mu \mathrm{m}$ is sufficient to achieve more than $90 \%$ absorption; the aspect ratio is moderate, enabling the use of standard photolithography techniques like direct laser writing for larger grating periods like the one of $8 \mu \mathrm{m}$ reported by Shimura et al. [9]. However, two factors favor the use of smaller periods: First the transversal coherence length $\xi$ has to be larger than the period $p_{1}$ of the phase grating, so a smaller period allows for the use of a larger source.

\footnotetext{
* Corresponding author.

E-mail addresses: Frieder.koch@kit.edu (F.J. Koch), juergen.mohr@kit.edu (J. Mohr).
} 
Transmission

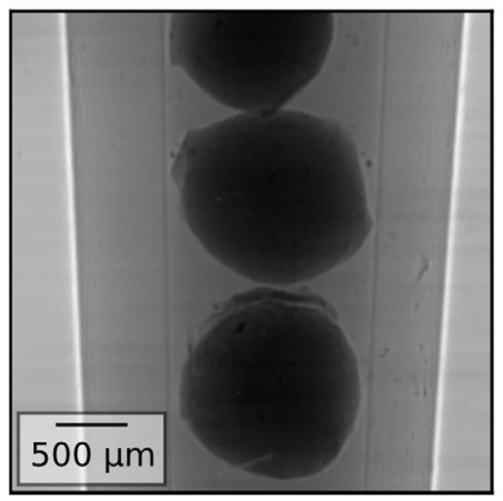

Differential phase

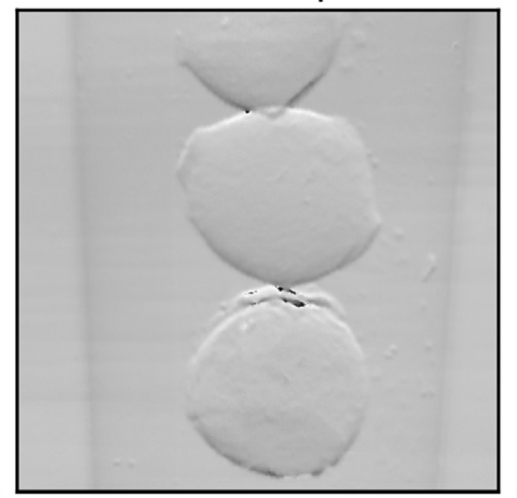

Dark field

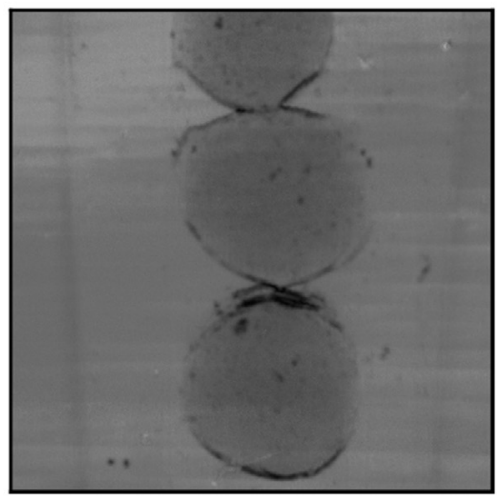

Fig. 1. Transmission, differential phase and dark field image of the polymer microspheres.

Second, the sensitivity of a grating based phase contrast setup, expressed by the smallest detectable refraction angle $\alpha_{\min }$, directly depends on the period of the self-image in the detector plane $p_{2}[15]$ :

$\alpha_{\min }=\frac{p_{2}}{2 \pi d} \frac{2}{V \sqrt{N}}$,

where $d$ is the distance between phase and analyzer grating, $V$ is the visibility of the self-image and $N$ is the number of photons detected in a frame. Hence the sensitivity can be increased by choosing a smaller grating period, which ultimately leads to the necessity of high aspect ratio fabrication techniques such as Silicon etching [16] or X-ray lithography [17] also for low energies.

Another characteristic of the gratings becomes important at low energies, namely the transmission of the grating substrates. The commonly used substrate material for high aspect ratio structures, silicon, should only have a thickness of about $8 \mu \mathrm{m}$ to ensure a transmission of $90 \%$ at $8 \mathrm{keV}$. As these thicknesses are impractical, one has to shift to alternative substrates made of lighter materials. This is possible in grating fabrication with X-ray lithography, which has been shown to work with graphite or Polyimide (PI) substrates [18]. In this paper, we present the implementation of a two-grating interferometer with $2.4 \mu \mathrm{m}$ period gratings on PI substrates, operating at $8.3 \mathrm{keV}$ at the beamline IMX of the Brazilian Synchrotron Light Laboratory. We show imaging results from a set of polymer microspheres filled with Fe nanoparticles, which give rise to a dark field signal.

\section{Experimental setup}

A multilayer monochromator was used to select a photon energy of $8.3 \mathrm{keV}$ from the bending magnet source at the IMX beamline. The source size is approximately $391 \mu \mathrm{m} \times 97 \mu \mathrm{m}$ (horizontal $\times$ vertical), and the phase grating was placed $17 \mathrm{~m}$ downstream of the source. The grating lines were oriented horizontally to make use of the smaller vertical source size and enable the use of a two-grating interferometer. The phase grating G1 had a period of $2.4 \mu \mathrm{m}$, with $\pi / 2$ phase shifting lines that were made from SU-8 with a thickness of $10 \mu \mathrm{m}$ to ensure high transmission. It was fabricated on a $1 \mathrm{~mm}$ thick DuPont Vespel ${ }^{\mathrm{TM}}$ substrate using a freeze drying process which enabled the fabrication of a line grating without supporting structures $[18,19]$. The absorption grating G2 had the same period, with absorbing lines of ca. $30 \mu \mathrm{m} \mathrm{Au}$, which leads to an absorption of over $99 \%$ of photons. This comparatively large thickness was chosen to make the grating suitable also for higher energies accessible at the IMX beamline. The substrate used was a PI foil with a thickness of $125 \mu \mathrm{m}$. Together, the substrates lead to a transmission of $43 \%$ at $8.3 \mathrm{keV}$, which could be improved to $83 \%$ by only using PI foils as substrates. Both the PI plate and foil are very stable and do not require special attention during handling, like thin membranes do. The inter-grating distance was set to $97 \mathrm{~mm}$, corresponding to the 5th fractional Talbot distance; the sample was placed $135 \mathrm{~mm}$ upstream of G1. Detection was done using a pco.2000 camera mounted behind a microscope objective and scintillator, yielding an effective pixel size of $1.64 \mu \mathrm{m}$ and a field of view of $3.4 \times 3.4 \mathrm{~mm}^{2}$. Assuming the same visibility and number of photons, this setup allows for an increase in sensitivity of a factor of 2.4 compared to the setup presented by Shimura et al. (distance G1-G2 of $135 \mathrm{~mm}$, absorption grating period $8 \mu \mathrm{m}$ [9]), while requiring less spatial coherence.

The phase grating was stepped over one period in eight steps, and the resulting phase stepping curve was analyzed using a discrete Fourier transform [20]. From the transformed stepping curve, we extract the zeroth term $A_{O}$ and the first term $A_{\boldsymbol{I}} \exp (i \varphi)$. The visibility $V$ is then calculated as $V=2 A_{1} / A_{0}$. Using an exposure time of $30 \mathrm{~s}$ per step, the mean visibility over the whole field of view was $40 \%$, mostly limited by setup instability and grating imperfections. The comparatively long exposure time is explained by the small flux after the monochromator at this energy. The sensitivity as determined from the standard deviation of the differential phase signal in a region of interest without sample was approximately $165 \mathrm{nrad}$. For applications that require higher sensitivity, this could be increased by choosing a larger inter-grating distance, although it is ultimately limited by the spatial coherence of the beam.

\section{Results and discussion}

Due to the low photon energy, the interferometer is mainly suited to the investigation of light and thin samples. We employed it to the study of magnetic beads, more precisely cellulose microspheres with embedded $\gamma-\mathrm{Fe}_{2} \mathrm{O}_{3}$-nanoparticles. The spheres themselves were suspended in ethyl alcohol in a capillary. The individual nanoparticles were coated with an oleate layer and had an average core size of $11 \mathrm{~nm}$, determined by transmission electron microscopy and X-ray diffraction data, and were thus much smaller than the systems spatial resolution. The concentration of the nanoparticles within the cellulose microspheres was 5 wt\%. For details about the preparation and characterization of the samples, please refer to the Supplemental material. Fig. 1 shows the three images obtained from the X-ray interferometer. The spheroid shape is visible in all three modalities, and the edges of the capillary show strong propagation based edge enhancement in the transmission image. It is also striking that the inner wall of the capillary is only visible in the transmission image. This is due to the one-dimensional grating setup, which is only sensitive to scattering and phase gradients in the direction perpendicular to the grating lines. The inner walls are nearly vertical, which means they give rise to both scattering and phase shift in the horizontal direction. The outer walls are slightly more inclined and, more importantly, present a much larger jump in refractive index (polymer-air interface instead of 

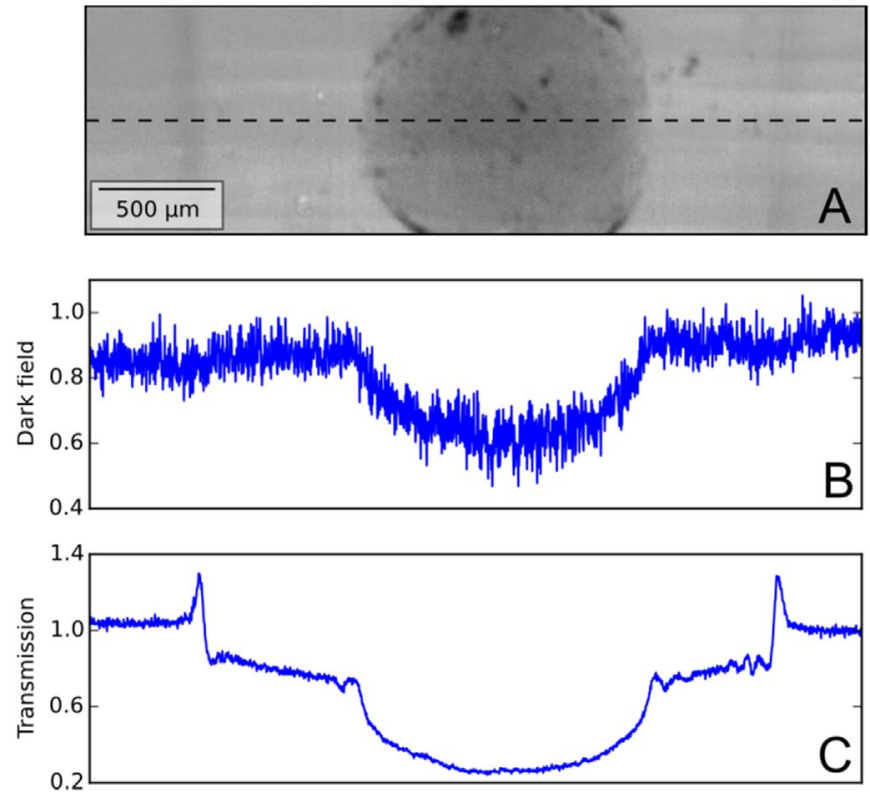

Fig. 2. A: section of the dark field signal shown in Fig. 1; B: line plot along the dashed line in the upper part. The dark field signal is not constant within the area of the sphere; C: transmission signal along the same line, it shows a similar behavior inside the microsphere as the dark field signal.

polymer-ethanol), they can thus be seen faintly in both the differential phase and the dark field. The capillary itself appears slightly darker than the background in the differential phase, which is due do its conical shape, which introduces a wedge-like phase shift.

The inside of the microspheres shows a rather strong dark field signal, i.e. a reduction in visibility. Due to their spherical shape, they introduce a non-negligible lens term to the wavefront phase, which is known to produce a dark field signal [21]. However, the dark field signal introduced by this effect should be constant over the whole region inside the sphere, which is not the case here. The magnitude of the dark field and transmission signal inside the lowest microsphere in the field of view is plotted in Fig. 2. The dark field signal inside the sphere shows a similar behavior as the transmission signal, indicating that it is indeed the material inside the sphere that produces the scattering, and the signal is not an artefact. The cause is instead most likely the $\mathrm{Fe}$ nanoparticles inside the spheres, which scatter the incoming X-rays. Fe incorporated in the polymer would be expected to contribute mainly to absorption, while the nanostructured particles produce both absorption and scattering.

\section{Conclusion}

A two grating interferometer at the IMX beamline of the Brazilian Synchrotron Light Laboratory, operating at a monochromatic energy of $8.3 \mathrm{keV}$ was established and tested. Grating interferometry at such low imaging energies is feasible with reasonable exposure times only when using gratings on low absorbing substrates, which can be produced by $\mathrm{X}$-ray lithography with small grating periods. We were able to achieve a visibility of $40 \%$, and used the interferometer to image polymer microspheres filled with $\gamma-\mathrm{Fe}_{2} \mathrm{O}_{3}$ nanoparticles, which produced a strong scattering signal and could therefore be detected as a loss in the visibility. The low imaging energy is especially interesting for light samples, such as polymers or biological specimen, which show poor contrast at higher energies. In future experiments, the transmission could be further improved by only using PI foils as substrates.

For further information about the magnetic bead samples, i.e. their preparation and characterization, please refer to the information given in the Supplemental material.

\section{Acknowledgements}

The authors acknowledge the support of the Karlsruhe School of Optics and Photonics (KSOP) and the Karlsruhe Nano Micro Facility (KNMF). The Brazilian Synchrotron Light Laboratory is acknowledged for providing beam time.

\section{Appendix A. Supplementary material}

Supplementary data associated with this article can be found in the online version at doi:10.1016/j.optcom.2017.02.055.

\section{References}

[1] F. Pfeiffer, T. Weitkamp, O. Bunk, C. David, Phase retrieval and differential phasecontrast imaging with low-brilliance X-ray sources, Nat. Phys. 2 (2006) 258-261. http://dx.doi.org/10.1038/nphys265.

[2] A. Momose, S. Kawamoto, I. Koyama, Y. Hamaishi, K. Takai, Y. Suzuki, Demonstration of $\mathrm{x}$-ray Talbot interferometry, Jpn. J. Appl. Phys. Part 2 Lett. 42 (2003) L866-L868. http://dx.doi.org/10.1143/JJAP.42.L866.

[3] A. Momose, T. Takeda, A. Yoneyama, I. Koyama, Y. Itai, Phase-contrast X-ray imaging using an X-ray interferometer for biological imaging, Anal. Sci. 17 (2001) $1-4$.

[4] A. Yaroshenko, F.G. Meinel, M. Bech, A. Tapfer, A. Velroyen, S. Schleede, S.D. Auweter, A. Bohla, A.Ö. Yildirim, K. Nikolaou, F. Bamberg, O. Eickelberg, M.F. Reiser, F. Pfeiffer, Pulmonary emphysema diagnosis with a preclinical smallanimal X-ray dark-field scatter-contrast scanner, Radiology 269 (2013) 427-433. http://dx.doi.org/10.1148/radiol.13122413.

[5] M. Stampanoni, Z. Wang, T. Thüring, C. David, E. Roessl, M. Trippel, R.A. KubikHuch, G. Singer, M.K. Hohl, N. Hauser, The first analysis and clinical evaluation of native breast tissue using differential phase-contrast mammography, Invest. Radiol. 46 (2011) 801-806. http://dx.doi.org/10.1097/RLI.0b013e31822a585f.

[6] H. Einarsdóttir, M.J. Emerson, L.H. Clemmensen, K. Scherer, K. Willer, M. Bech, R. Larsen, B.K. Ersbøll, F. Pfeiffer, Novelty detection of foreign objects in food using multi-modal X-ray imaging, Food Control 67 (2016), 2016, pp. 39-47. http://dx.doi.org/10.1016/j.foodcont.2016.02.023.

[7] F. Prade, K. Fischer, D. Heinz, P. Meyer, J. Mohr, F. Pfeiffer, Time resolved X-ray dark-field tomography revealing water transport in a fresh cement sample, Sci. Rep. 6 (2016) 29108. http://dx.doi.org/10.1038/srep29108.

[8] M. Ruiz-Yaniz, F. Koch, I. Zanette, A. Rack, P. Meyer, D. Kunka, A. Hipp, J. Mohr, F. Pfeiffer, X-ray grating interferometry at photon energies over $180 \mathrm{keV}$, Appl. Phys. Lett. 106 (2015) 151105. http://dx.doi.org/10.1063/1.4917293.

[9] T. Shimura, N. Morimoto, S. Fujino, T. Nagatomi, K. Oshima, J. Harada, K. Omote, N. Osaka, T. Hosoi, H. Watanabe, Hard x-ray phase contrast imaging using a tabletop Talbot-Lau interferometer with multiline embedded x-ray targets, Opt. Lett. 38 (2013) 157-159. http://dx.doi.org/10.1364/OL.38.000157.

[10] M. Chabior, T. Donath, C. David, M. Schuster, C. Schroer, F. Pfeiffer, Signal-tonoise ratio in $\mathrm{x}$ ray dark-field imaging using a grating interferometer, J. Appl. Phys. 110 (2011) 53105. http://dx.doi.org/10.1063/1.3630051.

[11] B. Trimborn, P. Meyer, D. Kunka, M. Zuber, F. Albrecht, S. Kreuer, T. Volk, T. Baumbach, T. Koenig, Imaging properties of high aspect ratio absorption gratings for use in preclinical x-ray grating interferometry, Phys. Med. Biol. 61 (2015) 527-541. http://dx.doi.org/10.1088/0031-9155/61/2/527.

[12] V. Revol, C. Kottler, R. Kaufmann, U. Straumann, C. Urban, Noise analysis of grating-based x-ray differential phase contrast imaging, Rev. Sci. Instrum. 81 (2010) 73709. http://dx.doi.org/10.1063/1.3465334.

[13] B.L. Henke, E.M. Gullikson, J.C. Davis, X-Ray interactions: photoabsorption, scattering, transmission, and reflection at $E=50--30,000 \mathrm{eV}, \mathrm{Z}=1-92$, At. Data Nucl. Data Tables 54 (1993) 181-342. http://dx.doi.org/10.1006/ adnd.1993.1013.

[14] M. Strobl, General solution for quantitative dark-field contrast imaging with grating interferometers, Sci. Rep. 4 (2014) 7243. http://dx.doi.org/10.1038/srep07243.

[15] T. Thüring, S. Hämmerle, S. Weiss, J. Nüesch, J. Meiser, J. Mohr, C. David, M. Stampanoni, Compact hard X-ray grating interferometry for table top phase contrast micro CT, in: R.M. Nishikawa, B.R. Whiting (Eds.), SPIE Medical Imaging, International Society for Optics and Photonicseds. (2013), 2013, p. 866813. http:// dx.doi.org/10.1117/12.2006865.

[16] C. David, J. Bruder, T. Rohbeck, C. Grünzweig, C. Kottler, A. Diaz, O. Bunk, F. Pfeiffer, Fabrication of diffraction gratings for hard X-ray phase contrast imaging, Microelectron. Eng. 84 (2007) 1172-1177. http://dx.doi.org/10.1016/ j.mee.2007.01.151.

[17] P. Meyer, J. Schulz, Deep X-ray lithography, in: Micromanufacturing Engineering Technology, Elsevier, 2015, pp. 365-391. doi: 〈http://doi.org/10.1016/B978-0323-31149-6.00016-5).

[18] F.J. Koch, T.J. Schröter, D. Kunka, P. Meyer, J. Meiser, A. Faisal, M.I. Khalil, L. Birnbacher, M. Viermetz, M. Walter, J. Schulz, F. Pfeiffer, J. Mohr, Note: gratings on low absorbing substrates for x-ray phase contrast imaging, Rev. Sci. Instrum. 86 (2015) 126114. http://dx.doi.org/10.1063/1.4939055.

[19] F. Koch, F. Marschall, J. Meiser, O. Márkus, A. Faisal, T. Schröter, P. Meyer, D. Kunka, A. Last, J. Mohr, Increasing the aperture of x-ray mosaic lenses by freeze drying, J. Micromech. Microeng. 25 (2015) 75015. http://dx.doi.org/10.1088/ 
0960-1317/25/7/075015.

[20] M. Engelhardt, C. Kottler, O. Bunk, C. David, C. Schroer, J. Baumann, M. Schuster, F. Pfeiffer, The fractional Talbot effect in differential $x$-ray phase-contrast imaging for extended and polychromatic x-ray sources, J. Microsc. 232 (2008) 145-157. http://dx.doi.org/10.1111/j.1365-2818.2008.02072x.

[21] J. Wolf, J.I. Sperl, F. Schaff, M. Schüttler, A. Yaroshenko, I. Zanette, J. Herzen, F. Pfeiffer, Lens-term- and edge-effect in X-ray grating interferometry, Biomed. Opt. Express 6 (2015) 4812. http://dx.doi.org/10.1364/BOE.6.004812. 